\title{
Titanate nanowire scaffolds decorated with anatase nanocrystals show good protein adsorption and low cell adhesion capacity
}

This article was published in the following Dove Press journal:

International Journal of Nanomedicine

7 February 2013

Number of times this article has been viewed

\author{
Xianglong Ding' \\ Xiaoqin Yang ${ }^{2}$ \\ Lei Zhou' \\ Haibin Lu' \\ Shaobing $\mathrm{Li}^{\prime}$ \\ Yan Gao' \\ Chunhua Lai' \\ Ying Jiang' \\ 'Center of Oral Implantology, \\ ${ }^{2}$ Department of Oral and Maxillofacial \\ Surgery, Guangdong Provincial \\ Stomatological Hospital, Southern \\ Medical University, Guangzhou, \\ People's Republic of China
}

Background and methods: In this report, layered microporous titanate nanowire scaffolds (TiNWs) were constructed via a hydrothermal route and then decorated with anatase nanocrystals (ANs@TiNWs) by immersion in $\mathrm{TiCl}_{4}$ solution. The diameter and specific surface area of the ANs@TiNWs was measured.The TiNWs and ANs@TiNWs were then compared for their ability to adsorb protein and adhere to MG63 cells.

Results: The diameter and specific surface area of the ANs@TiNWs were significantly larger than forTiNWs, and the ANs@TiNWs had an enhanced protein-adsorbing effect. It was found that the MG63 cells were less able to adhere to the flat titanium substrate than the TiNWs and ANs@TiNWs, and that this cell-repellant ability was greater with ANs@TiNWs. Other MG63 cell functions, proliferation in particular, were also inhibited by ANs@TiNWs.

Conclusion:ANs@TiNWs show a high protein adsorption and cell-repellant capacity which would be useful in drug delivery.

Keywords: protein adsorption, antiadhesion, nanotopography, drug delivery

\section{Introduction}

Desirable characteristics in a drug delivery system include a fully controlled drug release profile, and freedom from cellular adhesion and proliferation that would eventually lead to biofouling, impedance, blocking of pores, and general dysfunction of the delivery system. ${ }^{1,2}$ The design of effective structures protected from adhesion by mammalian cells has been a long-standing challenge. ${ }^{3}$ Relatively few studies have explored the use of nanostructures for preventing mammalian cell adhesion and survival in drug delivery systems. ${ }^{2,4}$ Further, the low protein adsorption onto nanostructures seen in previous studies indicates that it is difficult to load protein into drug delivery systems. A successful protein drug carrier should have a high protein adsorption capacity, because this assists loading of the protein drugs and their sustained release. ${ }^{5}$

Several recent studies have indicated that the response of cells coming into contact with the surface of a biomaterial depends mainly on spacing at the nanolevel. A study by Zhao et al showed that core-shell titanium carbide/carbon quasi-aligned nanowire arrays created by a thermochemical process inhibit adhesion of cells and have a relatively low protein adsorption capacity. ${ }^{4}$ An increase in protein adsorption is to be expected, given the increased specific surface area of the nanowire. Another study by Liu et al suggests that nanowire decorated with nanocrystals has an increased diameter and specific surface area, ${ }^{6}$ and recent work by Kim et al has shown that cell survival decreases as the diameter of the nanorod is increased from $30 \mathrm{~nm}$ to $400 \mathrm{~nm}$. Therefore, we hypothesized that nanocrystal-decorated nanowire arrays with a larger 
diameter and specific surface area may have a higher capacity for protein adsorption and a low capacity for cell adhesion.

Anatase coating is widely used on the surface of dental and orthopedic implants because of its good mechanical properties, chemical stability, and good biocompatibility. ${ }^{8}$ In this work, titanate nanowire scaffolds (TiNWs) were fabricated via a hydrothermal reaction and then immersed in $\mathrm{TiCl}_{4}$ to yield anatase nanocrystals (ANs@TiNWs)with an increased diameter and specific surface area. Furthermore, the influence of titanate nanowire scaffolds decorated withANs@TiNWs on protein adsorption and MG63 cell adhesion and proliferation was investigated to determine whetherANs@TiNWs are suitable for potential use as drug delivery systems, biosensors, and bioelectronic devices.

\section{Materials and methods} Preparation ofTiNWs and ANs@TiNWs

The TiNWs was prepared according to a previously described method. ${ }^{9}$ Before synthesis, titanium discs $15 \mathrm{~mm}$ in diameter $\times 1.5 \mathrm{~mm}$ thick were cut from commercially pure titanium plates (grade 2, Tengxing Metal Materials, Shenzhen, People's Republic of China). All titanium surfaces were ground with silicon carbide sandpaper of 280, 360, 400, 600,800 , and 1000 grit in series. The titanium discs were then immersed in $10 \mathrm{~mL}$ of acetone (Fuyu, Tianjin, People's Republic of China) at room temperature, ultrasonicated for 20 minutes, ultrasonicated for a further 20 minutes in $10 \mathrm{~mL}$ of absolute ethylalcohol (Fuyu), and then rinsed with deionized water. The titanium substrate was then placed in a sealed Teflon-lined vessel containing $10 \mathrm{~mL}$ of $1.0 \mathrm{~mol} / \mathrm{L} \mathrm{NaOH}$ solution. Next, the vessel was sealed and hydrothermally heated at $220^{\circ} \mathrm{C}$ for 8 hours. Treated titanium substrates covered with the titanate nanowire matrix were then collected, rinsed with a large amount of deionized water, and dried in air. Following this, the titanate nanowire matrix was placed in $100 \mathrm{~mL}$ of $\mathrm{TiCl}_{4}$ solution and kept there at $50^{\circ} \mathrm{C}$ for 0,2 , $4,6,8$, and 12 hours to allow anatase nanocrystals to grow around the periphery of the nanowires. The $\mathrm{TiCl}_{4}$ solution was prepared by mixing $0.09 \mathrm{~mL}$ of $\mathrm{TiCl}_{4}$ (Sigma-Aldrich, St Louis, MO, USA) with $0.4 \mathrm{~mL}$ of concentrated $\mathrm{HCl}$ solution (36.5\%-38\% by weight, Fuyu), followed by addition of deionized water to reach a final volume of $100 \mathrm{~mL} .{ }^{6}$ All samples were collected, rinsed with deionized water, and cleaned by autoclaving before cell plating.

\section{Surface characterization}

The morphology of each sample was analyzed using field emission scanning electron microscopy (1530VP, LEO Inc,
Oberkochen, Germany) and scanning electron microscopy (SEM, S-3000, Hitachi, Tokyo, Japan). The structure of each study sample was analyzed by transmission electron microscopy (TEM, Tecnai-10, Philips, Eindhoven, The Netherlands) at an acceleration voltage of $200 \mathrm{kV}$. The phase components of the samples from each synthesis step was investigated by X-ray diffraction (D8 Advance, Bruker, Germany) with $\mathrm{Cu} \mathrm{K} \alpha$ radiation $(\lambda=0.15418 \mathrm{~nm}, 40 \mathrm{kV}, 40 \mathrm{~mA})$ from 5 to 90 degrees at a scanning speed of 0.2 degrees per second. Water contact angle measurements were carried out using the sessile drop technique (OCA40 Micro, DataPhysics, Filderstadt, Germany) at room temperature. Nitrogen adsorption/desorption isotherms of the nanowires were measured using an ASAP 2000 (Micrometrics, Cumming, GA, USA) at $77 \mathrm{~K}$. Prior to these measurements, the nanowires were degassed at $353 \mathrm{~K}$ overnight.

\section{Measurement of protein adsorption}

The ability of the TiNWs and ANs@TiNWs to adsorb a model protein (bovine serum albumin, fraction V (Sigma-Aldrich)) was compared with the protein-adsorptive capacity of a flat titanium wafer used as a control. First, $300 \mu \mathrm{L}$ of protein solution ( $1 \mathrm{mg}$ protein per $\mathrm{mL}$ saline) was spread over each specimen using a pipette. After incubation for 1, 6, 12, and 24 hours under sterile humidified conditions at $37^{\circ} \mathrm{C}$, all nonadherent protein was removed, and the remaining solution was mixed with micro bicinchoninic acid (Sigma-Aldrich) at $37^{\circ} \mathrm{C}$ for 30 minutes. The protein content of the solution was quantified using a microplate reader (Model 680, Bio-Rad, Hercules, CA, USA) at $562 \mathrm{~nm}$.

\section{Cell culture}

A human MG63 osteosarcoma cell line was cultured in Dulbecco's modified Eagle's medium (Hyclone, Thermo Fisher Scientific, Logan, UT, USA) supplemented with 10\% fetal bovine serum (Hyclone, Thermo Fisher Scientific) and antibiotics (penicillin $100 \mathrm{U} / \mathrm{mL}$, streptomycin $100 \mathrm{mg}$ / $\mathrm{mL}$ ) at $37^{\circ} \mathrm{C}$ in $5 \% \mathrm{CO}_{2}$. At $80 \%$ confluence, the cells were detached using $0.25 \%$ trypsin 1 mM EDTA-4 Na and seeded onto the ANs@TiNWs in 24-well plates at a density of $2.4 \times 10^{4}$ cells $\mathrm{cm}^{-2}$. The culture medium was replaced on alternate days.

\section{Cell morphology assay}

SEM was used to analyze the cell morphology 24 hours after the MG63 cells had been seeded onto the flat titanium, TiNWs, and ANs@TiNWs. The culture medium with unattached cells was carefully removed from the wells by pipette, 
and the titanium, TiNWs, and ANs@TiNWs were then rinsed twice with phosphate-buffered solution. Cells on the samples were fixed with $2.5 \%$ glutaraldehyde and phosphate-buffered solution for one hour, and washed three times with phosphatebuffered solution for 5 minutes at room temperature. Each sample then underwent graded dehydration with $30 \%, 50 \%$, $70 \%, 90 \%$, and $100 \%$ pure ethyl alcohol for 10 minutes at room temperature, after which the samples were dried for analysis by SEM.

\section{Immunofluorescent staining}

Cytoskeletal changes in the MG63 cells were observed using an immunofluorescent staining technique. ${ }^{10}$ After incubation of the cells for 24 hours, the culture medium was gently removed from the well. The cells were washed with phosphate-buffered solution, fixed with $4 \%$ paraformaldehyde for 20 minutes, and then washed several times with phosphate-buffered solution. The fixed cells were treated with $0.1 \%$ Triton X-100 in phosphate-buffered solution and then blocked with $1.0 \%$ bovine serum albumin in phosphate-buffered solution. Actin cytoskeleton was stained with phalloidin conjugated to Alexa Fluor 488 dyes (Invitrogen, Carlsbad, CA, USA) and the nucleus was stained with Hoechst 33342 dye (Invitrogen). The stained cells were observed under a fluorescence microscope (IX51, Olympus, Tokyo, Japan).

\section{Cell adhesion assay}

Cell attachment was initially evaluated by measuring the amount of cells attached to the titanium substrates after 30 minutes and 4 hours of incubation, as described previously. ${ }^{10}$ At each prescribed time point, nonadherent cells were removed by gentle rinsing with phosphate-buffered solution. Adherent cells were fixed with 4\% paraformaldehyde for 30 minutes, and then stained with fluorescent Hoechst 33342 dye for 5 minutes. Cell adhesion was evaluated by counting the number of stained nuclei on each sheet seen in the fluorescent microscopic images $(100 \times$ over an area of $1800 \times 1350 \mu \mathrm{m}^{2}$ ). Values representing the mean and the standard error of the number of attached cells were calculated from ten different random fields in each disc using Image-Pro Plus $6.0(n=3$, total of 30 points for each group).

\section{Cell proliferation assay}

Cell proliferation was determined in terms of cell density on culture days 1 and 4 using tetrazolium salt-based colorimetry (MTS, Promega, Madison, WI, USA).${ }^{10}$ At each prescribed time point, the specimens were gently rinsed three times with phosphate-buffered solution and transferred to a new 24-well plate. Next, $500 \mu \mathrm{L}$ of Dulbecco's modified Eagle's medium was added to each well, and incubated with $100 \mu \mathrm{L}$ of MTS reagent at $37^{\circ} \mathrm{C}$ for 4 hours. The amount of formazan product was measured using a microplate reader at $490 \mathrm{~nm}$.

\section{Statistical analysis}

All the experiments described above were performed in triplicate. One-way analysis of variance was used to assess the statistical significance of differences between the titanium groups. All statistical analyses were performed using SPSS version 16.0 software (SPSS Inc, Chicago, IL, USA). Results were considered to be statistically significant at $P<0.05$.

\section{Results \\ Surface characterization of TiNWs and ANs@TiNWs}

Representative field emission scanning electron microscopic and TEM images of TiNWs and ANs@TiNWs are shown in Figure 1. After hydrothermal reaction in sodium hydroxide at $220^{\circ} \mathrm{C}$, the titanium surface formed TiNWs characterized by interconnected macropores 5-20 $\mu \mathrm{m}$ in diameter and composed of many nanowires bundled together (Figure 1A and B). Figure 1C shows nanowires 40-65 nm in diameter, and Figure S1 indicates that the nanowires
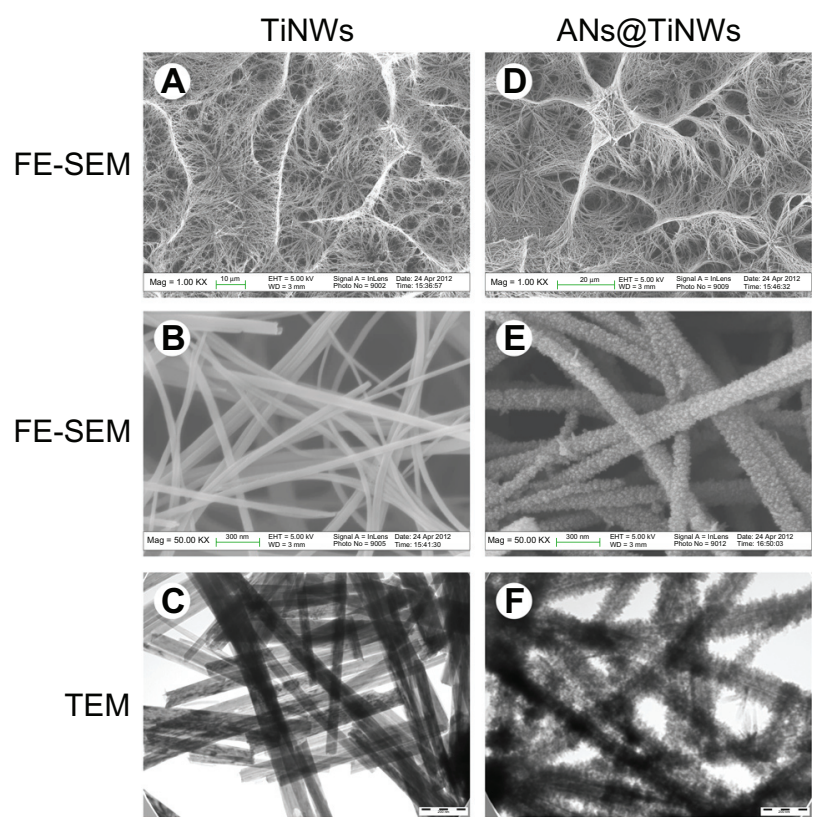

Figure I FE-SEM and TEM images of TiNWs and ANs@TiNWs formed by immersing the TiNWs in $\mathrm{TiCl}_{4}$ for 6 hours. (A) FE-SEM images of TiNWs (I000X), (B) FE-SEM images of TiNWs $(50,000 \times)$, (C) TEM images of TiNWs, (D) FE-SEM images of ANs@TiNWs (I000×), (E) FE-SEM images of ANs@TiNWs $(50,000 \times)$, and (F) TEM images of ANs@TiNWs.

Abbreviations: ANs@TiNWs, titanate nanowire scaffolds decorated with anatase; FE-SEM, field emission scanning electron microscopy; TEM, transmission electron microscopy; TiNWs, titanate nanowire scaffolds. 
Table I Diameters and specific surface areas of microporous titanate nanowire scaffolds and microporous titanate nanowire scaffolds decorated with anatase crystals

\begin{tabular}{lllllll}
\hline Time, hours & $\mathbf{0}$ & $\mathbf{2}$ & $\mathbf{4}$ & $\mathbf{6}$ & $\mathbf{8}$ & $\mathbf{1 2}$ \\
\hline Diameter $(\mathrm{nm})^{*}$ & $51.27 \pm 7.24$ & $104.3 \pm 14.89$ & $142.78 \pm 10.01$ & $152.36 \pm 16.97$ & $165.06 \pm 10.59$ & $189.35 \pm 9.66$ \\
Specific surface area $\left(\mathrm{m}^{2} / \mathrm{g}\right)^{*}$ & $15.43 \pm 0.25$ & $22.07 \pm 0.35$ & $25.20 \pm 0.36$ & $29.33 \pm 0.40$ & $33.23 \pm 0.30$ & $35.93 \pm 0.35$ \\
\hline
\end{tabular}

Note: *Values are given as the mean \pm standard deviation.

were about $10-15 \mu \mathrm{m}$ in length. When the TiNWs were immersed in $\mathrm{TiCl}_{4}$ solution, their surfaces became covered by anatase nanocrystals, ie, ANs@TiNWs (Figure 1D-F). After 2 hours in $\mathrm{TiCl}_{4}$ solution, the anatase nanocrystals were observed to be well distributed on the surface of the TiNWs. The anatase nanocrystals grew larger with increasing time in $\mathrm{TiCl}_{4}$ solution, with a corresponding increase in the diameter and specific surface area of the ANs@TiNWs (Table 1 and Figure S2).

Figure 2 shows the $\mathrm{x}$-ray diffraction patterns from the flat titanium, TiNWs, and ANs@TiNWs. The pattern from the TiNW array yielded via the alkali hydrothermal growth process could be indexed to body-centered orthorhombic $\mathrm{Na}_{2} \mathrm{Ti}_{2} \mathrm{O}_{5} \cdot \mathrm{H}_{2} \mathrm{O}$, which is made up of two-dimensional sheets composed of edge-sharing $\mathrm{TiO}_{6}$ octahedra (Figure 2B). ${ }^{6} \mathrm{After}$

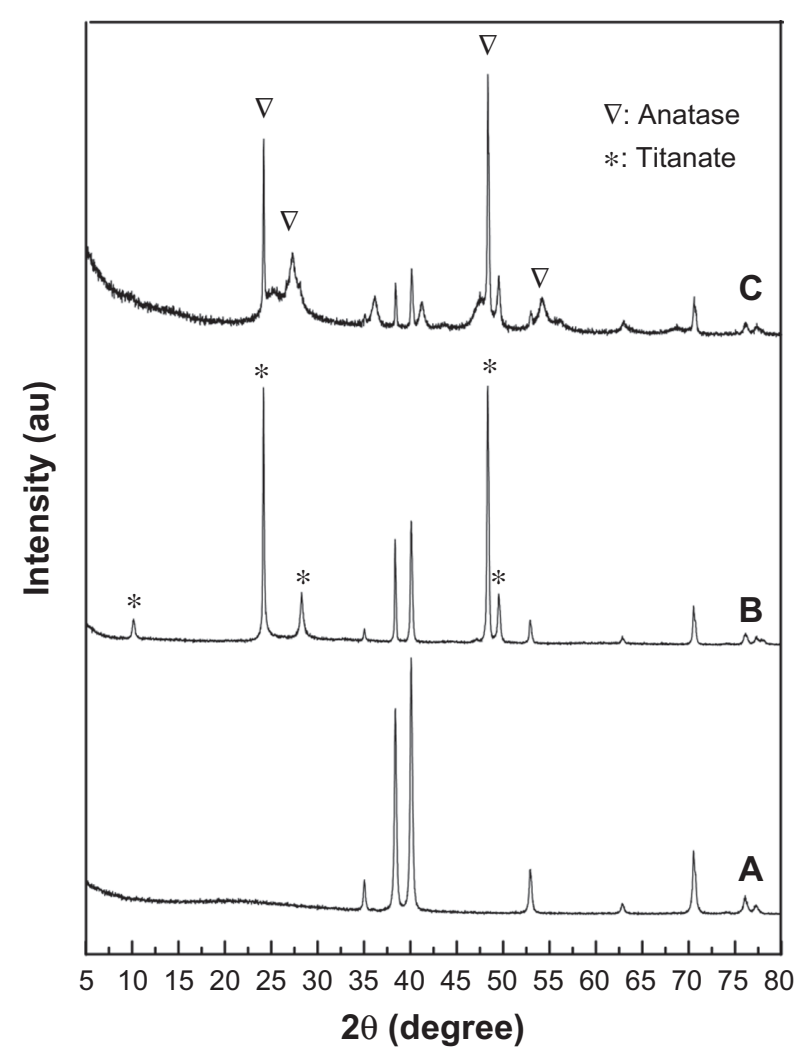

Figure 2 X-ray diffraction patterns for (A) flat titanium, (B) titanate nanowire scaffolds, and (C) titanate nanowire scaffolds decorated with anatase. growth with anatase nanocrystals, well resolved anatase diffractions were clearly evident (Figure 2C).

Contact angle measurements are shown in Figure 3. The average contact angle of the flat titanium surface (control) was about 75 degrees.The TiNWs and ANs@TiNWs showed superhydrophilicity at a water contact angle of 0 degrees.

\section{Increased protein adsorptive capacity of ANs@TiNWs}

Protein adsorptive rates for flat titanium, TiNWs, and ANs@ TiNWs (formed by immersion of TiNWs in $\mathrm{TiCl}_{4}$ solution for different time periods, ie, 2, 4, 6, 8, and 12 hours) were analyzed using a bicinchoninic acid assay, and the results are shown in Figure 4. All the ANs@TiNWs groups showed a higher protein adsorption rate than the flat titanium control group and TiNW group regardless of incubation time, and the TiNWs showed no significant difference in protein adsorption rate compared with flat titanium.ANs@TiNWs incubated for 6 hours in $\mathrm{TiCl}_{4}$ solution had the highest protein adsorption rate of all the groups. The protein adsorptive rate of ANs@, TiNWs incubated for 6 hours reached 25\% after one hour of incubation, and approximately $67 \%$ after incubation for 6 hours. The protein adsorptive capacity of ANs@TiNWs decreased when they were immersed in $\mathrm{TiCl}_{4}$ solution for longer than 6 hours.

\section{Changes in cell morphology on exposure to TiNWs and ANs@TiNWs}

Figure 5 shows SEM images revealing the morphology of MG63 cells cultured on flat titanium, TiNWs, and ANs@, TiNWs. The ANs@TiNWs group immersed in $\mathrm{TiCl}_{4}$ solution for 6 hours showed the highest protein adsorption rate and therefore was used for all cell tests. The MG63 cells attached and spread well on the flat titanium wafer after 24 hours in culture, with some lamellipodia and filopodia stretching out to anchor onto the flat titanium wafer (Figure 5A). In contrast, there were few MG63 cells on the surfaces of the TiNWs and ANs@TiNWs. It was observed that the MG63 cells did not adhere to the underlying TiNW and ANs@TiNWs substrates, but penetrated and became fixed to the nanowires themselves 
$\mathrm{Ti}$

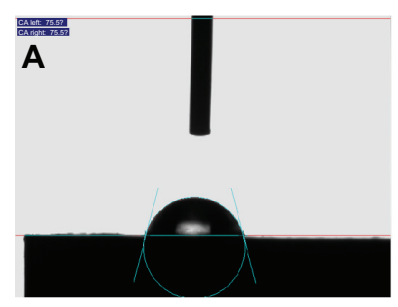

TiNWs

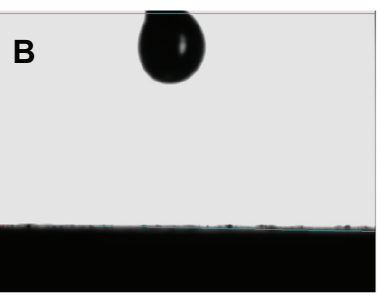

\section{ANs@TiNWs}

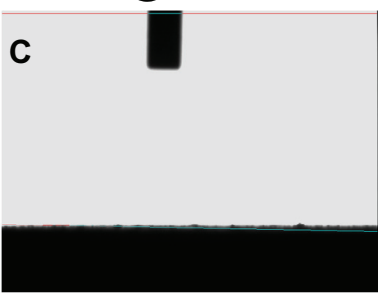

Figure 3 Water contact angles on (A) flat titanium, (B) TiNWs, and (C) ANs@TiNWs.

Abbreviations: ANs@TiNWs, titanate nanowire scaffolds decorated with anatase; TiNWs, titanate nanowire scaffolds; Ti, flat titanium.

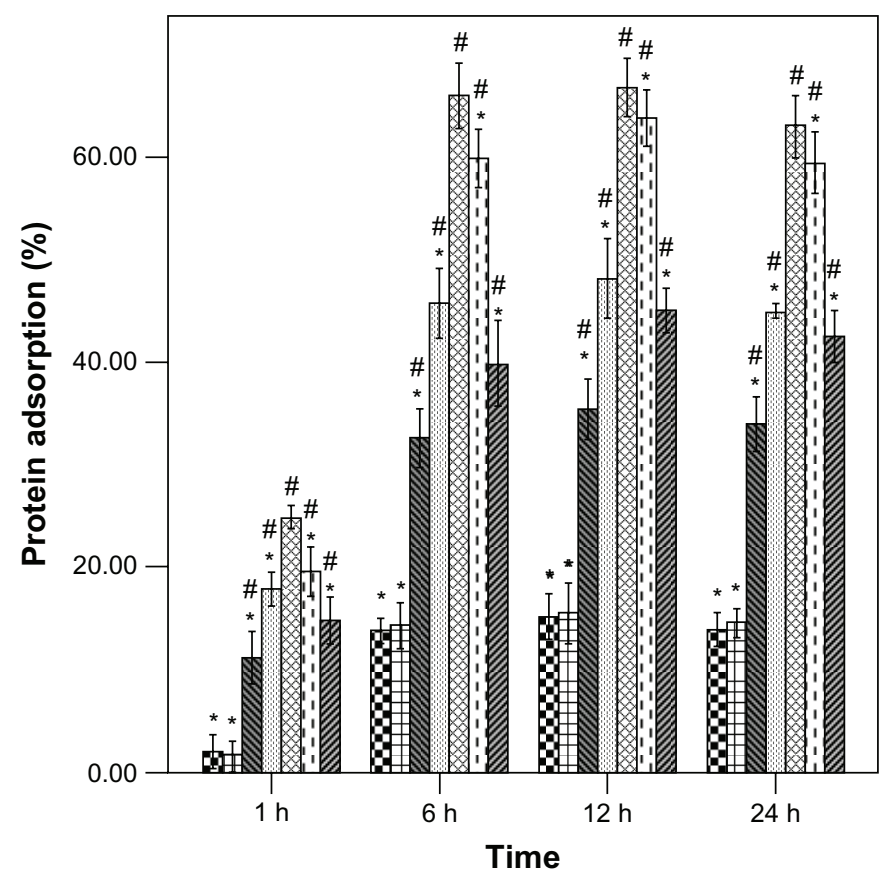

$\mathbf{Q T i}_{\mathrm{Ti}}$

再TiNWs

$\mathbb{N}$ ANs@TiNWs/2h

ANs@TiNWs/4h

网ANs@TiNWs/6h

团ANs@TiNWs/8h

ZMANs@TiNWs/12 h

Time

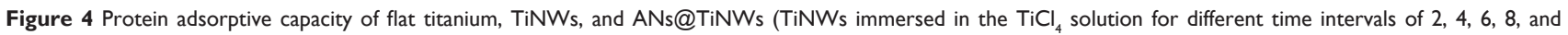
12 hours) assessed using a model protein.

Notes: *P $<0.05$ compared with the ANs@TiNWs incubated for 6 hours. ${ }^{\#}<0.05$ versus flat titanium.

Abbreviations: ANs@TiNWs, titanate nanowire scaffolds decorated with anatase; TiNWs, titanate nanowire scaffolds; Ti, flat titanium.
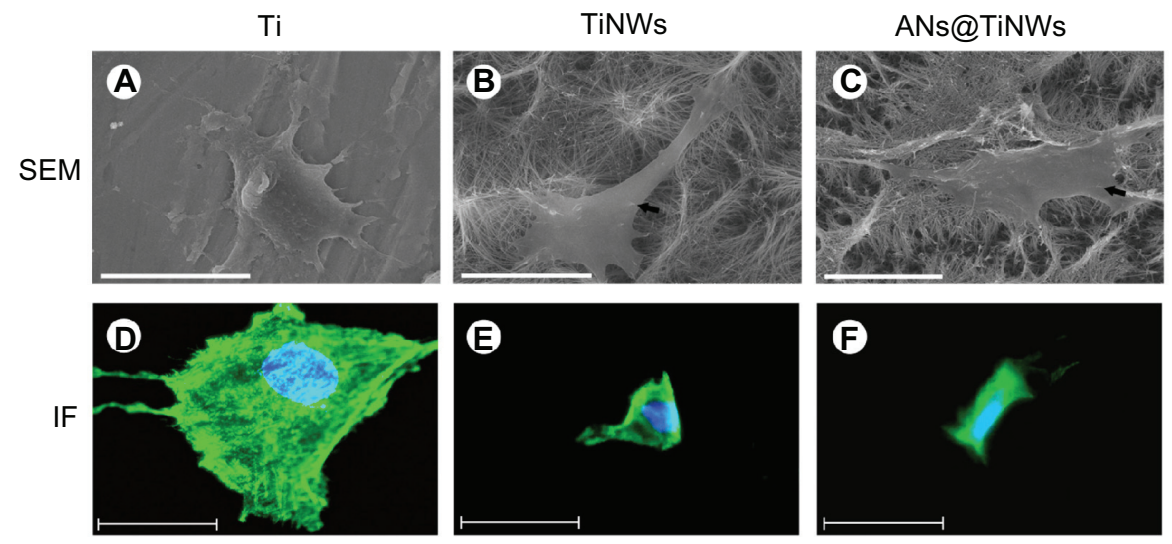

Figure 5 SEM and immunofluorescent staining micrographs of MG63 cells cultured on flat titanium, TiNWs, and ANs@TiNWs. (A) MG63 cells attach and spread well onto the flat titanium surface after 24 hours in culture. MG63 cells on TiNWs (B) and ANs@TiNWs (C) were penetrated and fixed by several nanowires (indicated by black arrows, scale bar $20 \mu \mathrm{m}$ ). (D-F) Immunofluorescent micrographs of MG63 cells stained for actin (green) and nuclei stained with Hoechst dye (blue).

Note: The cell spreading area is greatly reduced, and few stress fibers are observed in cells cultured on TiNWs and ANs@TiNWs. Scale bar $50 \mu \mathrm{m}$.

Abbreviations: ANs@TiNWs, titanate nanowire scaffolds decorated with anatase; IF, immunofluorescent staining; SEM, scanning electron microscopy; TiNWs, titanate nanowire scaffolds; Ti, flat titanium. 
(Figure 5B and $\mathrm{C}$, indicated by black arrows). Figure 5A-C shows that the MG63 cells fixed onto the TiNW and ANs@, TiNWs arrays did not expand as freely as they did on flat titanium.

MG63 cell adhesion and spreading on flat titanium, TiNWs, andANs@TiNWs was investigated by immunofluorescent staining. Fluorescence images of MG63 cells adhered onto the flat titanium, TiNW, and ANs@TiNWs substrates are shown in Figure 5D-F. The number of MG63 cells containing clear stress fibers was markedly greater on the flat titanium substrate. However, fewer stress fibers were observed, and the MG63 cells were poorly distributed on the TiNWs and ANs@TiNWs.

\section{Decreased cell adherence on TiNWs and ANs@TiNWs}

Initial cell adhesion on flat titanium, TiNWs, and ANs@ TiNWs was estimated by counting the number of MG63 cell nuclei stained with Hoechst dye, as shown in Figure 6. The results were calculated using software Image-Pro Plus 6.0 and are displayed by histogram in Figure 7. At the time points used in this study, the numbers of cells adherent on the TiNWs and ANs@TiNWs were significantly smaller than those adherent to flat titanium. The numbers of cells adherent to TiNWs and ANs@TiNWs were about 30\% and $24 \%$, respectively, less than those adherent to flat titanium after incubation for 30 minutes. After 4 hours of incubation, most of the cells seeded had attached to the surface of the flat
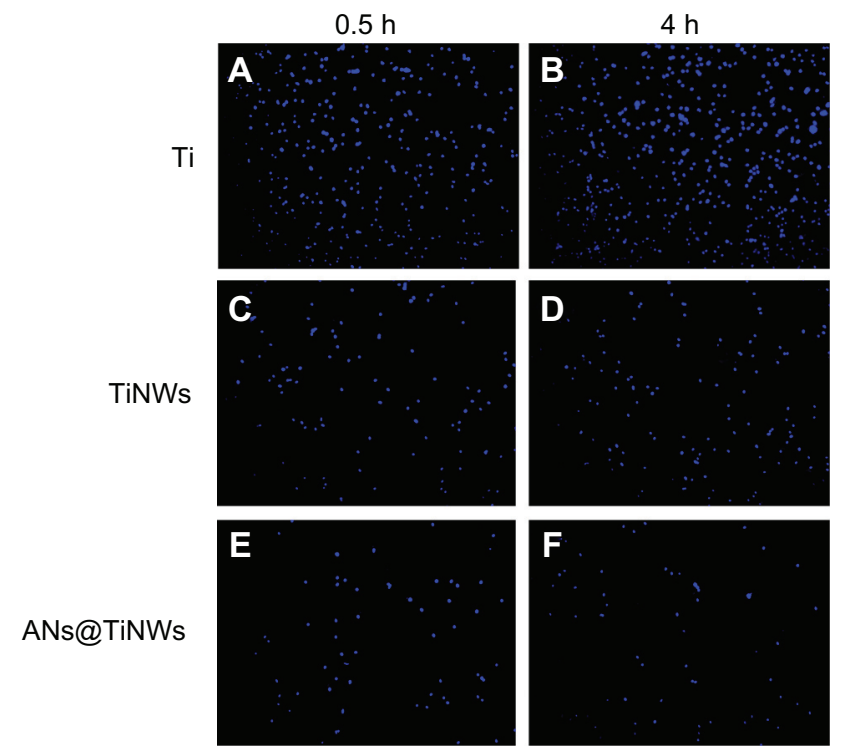

Figure 6 The number of MG63 cell nuclei stained with Hoechest dye on flat titanium, TiNWs, and ANs@TiNWs at $0.5 \mathrm{~h}(\mathbf{A}, \mathbf{C}$ and $\mathbf{E})$ and $4 \mathrm{~h}(\mathbf{B}, \mathbf{D}$ and F). Abbreviations: ANs@TiNWs, titanate nanowire scaffolds decorated with anatase; TiNWs, titanate nanowire scaffolds; Ti, flat titanium.

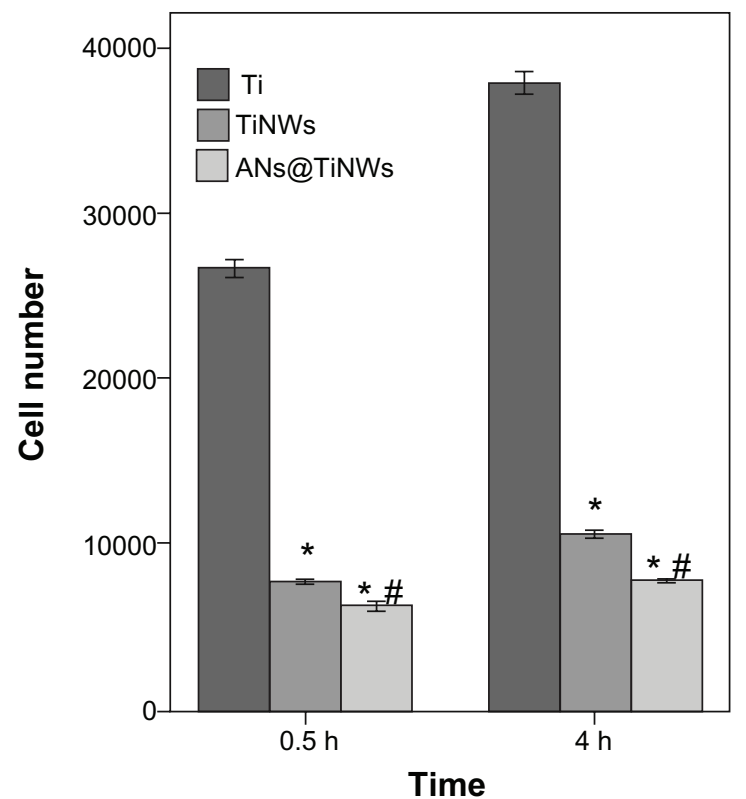

Figure 7 Mean ( \pm standard deviation) cell numbers calculated from Figure $6(n=3$, 10 different random fields of each disc).

Notes: $* P<0.05$ versus flat titanium group; ${ }^{*} P<0.05$ versus TiNW group.

Abbreviations: ANs@TiNWs, titanate nanowire scaffolds decorated with anatase; TiNWs, titanate nanowire scaffolds; Ti, flat titanium.

titanium wafer, with only about $25 \%$ and $18 \%$ of that amount attached to the TiNWs and ANs@TiNWs, respectively. Therefore,ANs@TiNWs have a greater ability than TiNWs to inhibit MG63 cell adhesion.

\section{Inhibition of proliferation of cells seeded onTiNWs and ANs@TiNWs}

Proliferation of the MG63 cells seeded onto the flat titanium, TiNWs, and ANs@TiNWs was measured using the MTS assay. Proliferation of cells cultured on TiNWs and ANs@TiNWs was reduced at days 1 and 4 compared with that on the flat titanium surface $(P<0.05)$. Moreover, less cell proliferation was observed on ANs@TiNWs than on TiNWs ( $P<0.05$, Figure 8$)$. The toxicity of the study materials was determined by cell morphology and MTS assay, which showed that cell death was not due to toxicity of materials dissolving from the nanowires (Figures S3 and S4).

\section{Discussion}

In this work, we fabricated layered microporous TiNW scaffolds via a simple hydrothermal route at low temperature, which we then decorated with anatase nanocrystals in $\mathrm{TiCl}_{4}$ solution to formANs@TiNWs.After the hydrothermal reaction, dense nanowires have grown on titanium and eventually self-assembled into three-dimensional macroporous scaffolds, 


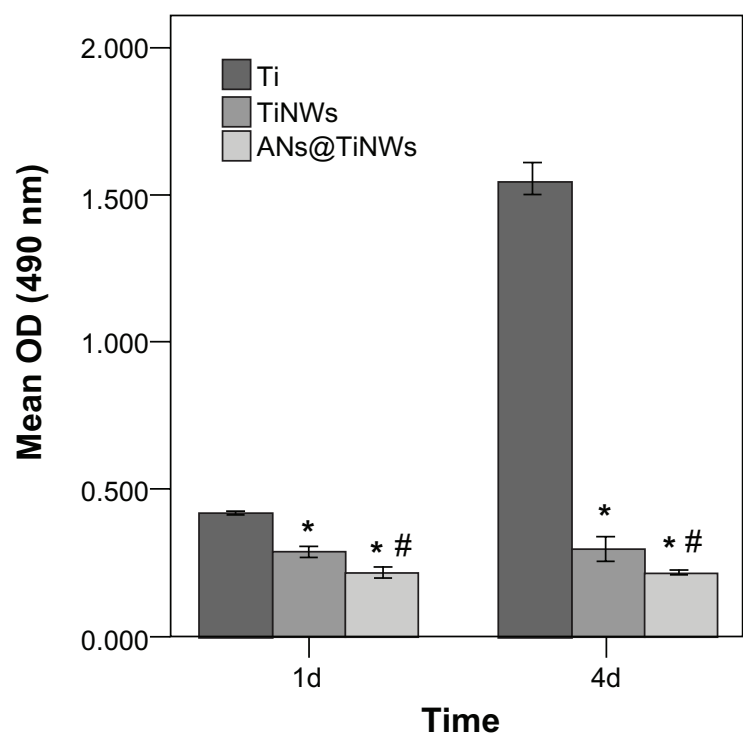

Figure 8 Proliferation of MG63 cells seeded onto flat titanium,TiNWs, and ANs@ TiNWs as measured by MTS assay.

Notes: The mean \pm standard deviation was calculated $(n=3)$. $* P<0.05$ versus flat titanium group; ${ }^{\#}<0.05$ versus TiNW group.

Abbreviations: ANs@TiNWs, titanate nanowire scaffolds decorated with anatase; TiNWs, titanate nanowire scaffolds; Ti, flat titanium; OD, optical density.

with a typical pore diameter of around 5-20 $\mu \mathrm{m}$. Nanowires as a novel one-dimensional nanomaterial have attracted increasing interest in recent years. Their unique optical, electronic, and catalytic properties suggest many promising applications in solar cells $\mathrm{s}^{11,12}$ and photocatalysis. ${ }^{6}$ Moreover, ANs@TiNWs have the unique properties of both nanowires and nanocrystals, making them interesting nanostructures with potential novel functions.

Our results show that the ANs@TiNWs group had a higher protein adsorption rate than that in the flat titanium group and the TiNW group, regardless of duration of incubation. It is speculated that this higher protein adsorption is due largely to the increase in specific surface area of the ANs@ TiNWs available for decorating with anatase nanocrystals. Furthermore, it was shown in this study that ANs@TiNWs incubated for 6 hours had the highest protein adsorption rate of all groups, with rates reaching 25\% in ANs@TiNWs incubated for one hour and 67\% in ANs@TiNWs incubated for 6 hours. Although the specific surface areas of the ANs@TiNWs incubated for 8 and 12 hours $\left(33.2 \mathrm{~m}^{2} / \mathrm{g}\right.$ and $35.9 \mathrm{~m}^{2} / \mathrm{g}$, respectively) are both larger than that of the ANs@ TiNWs incubated for 6 hours $\left(29.3 \mathrm{~m}^{2} / \mathrm{g}\right)$, the amounts of bovine serum albumin adsorbed $\left(67.2 \mathrm{kDa}^{13}\right)$ onto ANs@ TiNWs incubated for 8 and 12 hours were smaller than that adsorbed ontoANs@TiNWs incubated for 6 hours. This can be explained by the fact that as the diameter increases, the gap between the nanowires becomes narrower than the size of the protein, making it harder for protein to absorb onto the nanowires.

Interestingly, although the TiNWs had a larger specific surface area than the flat titanium wafer control, they did not show a corresponding increase in protein adsorption rate. A possible reason for this is that insertion of liquid into nanostructures is difficult, because the surface tension of the liquid needs to overcome the air trapped among the nanowires, ${ }^{2}$ leading to less protein being absorbed onto the outer sphere of the nanowires. The specific surface area of the ANs@TiNWs was markedly increased after being decorated with anatase nanocrystals, with a corresponding increase in protein adsorptive capacity, even though only the outer spheres of the ANs@TiNWs can be used. In this study, bovine serum albumin was used as a model protein, and antibiotics, chemotherapeutic drugs, steroids, hormones, and genes could also be used in this system for future research.

It is thought that surface wettability plays an important role in interactions between cells and biomaterials because it may impact cell behavior directly or indirectly by affecting protein adsorption. ${ }^{4}$ The contact angle of the water droplet with TiNWs and ANs@TiNWs was measured in our study, and was found to be 0 degrees, which is consistent with the findings of previous studies showing that the surfaces of both TiNWs and ANs@TiNWs are superhydrophilic. ${ }^{2,14}$ When a water droplet was added to TiNW or ANs@TiNWs, it was immediately and completely absorbed into the pores of the nanowires. This phenomenon can be attributed to the chemical structure of $\mathrm{Na}_{2} \mathrm{Ti}_{2} \mathrm{O}_{5} \cdot \mathrm{H}_{2} \mathrm{O}$. The hydroxyl and crystallized water in these nanotitanate structures can easily absorb water from the environment. ${ }^{14}$ This surface allows for complete wetting of the protein solution, potentially improving its drug-loading capability as well, ${ }^{2}$ although there are an increasing number of reports pointing to limited effects of hydrophilicity in determining the bioactivity of material surfaces, ${ }^{15,16}$ and our results support this view.

Furthermore, we found that adhesion of MG63 cells was greatly reduced on TiNWs and ANs@TiNWs compared with that on flat titanium, and that ANs@TiNWs had a greater inhibitory effect. Meanwhile, it was observed that few stress fibers were visible in cells on the TiNWs and ANs@TiNWs. Our SEM observations indicate that the lamellipodia and filopodia of MG63 cells were not able to attach onto TiNWs and ANs@TiNWs.

We speculate that there are three factors leading to limited cell attachment and spreading on the nanostructures used in this work. Firstly, focal adhesion is impaired because of 
the limited binding sites, which is caused by abundant gaps between nanowires. As we know, the deficient binding sites available for cells clustering integrins into focal adhesion complexes, will lead to decreased cell adhesion. ${ }^{3,17}$ In our study, we found that the gaps formed by paralleled and interlaced nanowires were varying from several nanometers to dozens of microns. As we know, cell interactions with extracellular surfaces are mediated by clustering of integrins into focal adhesion complexes in which the spacing between integrin ligands plays a key role in the intracellular signaling cascades occurring in the nucleus and activated in the cytoskeleton. ${ }^{18-21}$ For these reasons, there was decreased cell adhesion on TiNWs and ANs@TiNWs.

Secondly, apoptosis of cells caused by the penetration of nanowires is depended on the diameter of nanowires. According to the results of Kim et al, ${ }^{7}$ cell death occurred within a day on nanowires with a larger diameter (400 nm), whereas cells grown on nanowires with a smaller diameter $(30 \mathrm{~nm})$ remained alive for more than 5 days. In our study, the diameter of the nanowires was about 40-65 nm, while the nanowires decorated by the nanocrystals had a diameter of about 145-170 nm, suggesting that the cells may be penetrated to apoptosis by these nanowires. Therefore, the ANs@TiNWs with larger diameter showed lower cell adhesive capacity than TiNWs. In addition, it is possible that nanowires prevented the cell from coming into contact with the basal "planar" substrate, leading to reduced cellular adhesion to TiNWs and ANs@TiNWs, depending on the height and density of the nanowires. ${ }^{22}$ Moreover, proliferation of MG63 cells cultured on the TiNWs and ANs@TiNWs was reduced compared with that on the flat titanium wafer at days 1 and 4. Therefore, inhibition of MG63 cell proliferation may be attributed to less cell adhesion on TiNWs and ANs@TiNWs.

The relationship between protein adsorption ability and cell adhesion is another controversial topic. Many researchers believe protein adsorption onto biomaterial surfaces is the initial critical step that determines cell adhesion. Stem/osteoprogenitor cells attach to the implant surface via interaction of cell integrins with adsorbed proteins, the extent of which may consequently affect the spread, proliferation, and differentiation of cells. ${ }^{23-26}$ Osteoblast attachment on titanium surfaces in the presence of preadsorbed proteins was observed to be enhanced compared with osteoblast attachment on control titanium surfaces without preadsorbed proteins. ${ }^{27}$ However, ANs@TiNWs with high protein adsorption showed low MG63 cell adhesive capacity in our study, which may be due to the special nanostructure of the ANs@,
TiNWs, with their larger specific surface area favoring protein adsorption but limited binding sites available for adhesion of MG63 cells.

\section{Conclusion}

In summary, ANs@TiNWs have a large diameter and specific surface area, enabling good protein adsorption and limited cell adhesion. This unique nanostructure is useful from the perspective of drug delivery because we can easily achieve a high loading capacity for protein-based compounds while simultaneously maintaining good cell-repellant properties. Although several studies of drug loading in nanowire delivery systems have already been reported, to the authors' knowledge this is the first study describing the unique structure of ANs@TiNWs.

\section{Acknowledgments}

This work was supported by the National Natural Science Foundation of China (81170998).

\section{Disclosure}

The authors report no conflicts of interest in this work.

\section{References}

1. Voskerician G, Shive MS, Shawgo RS, et al. Biocompatibility and biofouling of MEMS drug delivery devices. Biomaterials. 2003;24: 1959-1967.

2. Brammer KS, Choi C, Oh S, et al. Antibiofouling, sustained antibiotic release by Si nanowire templates. Nano Lett. 2009;9:3570-3574.

3. Lee J, Chu BH, Chen KH, Ren F, Lele TP. Randomly oriented, upright $\mathrm{SiO}_{2}$ coated nanorods for reduced adhesion of mammalian cells. Biomaterials. 2009;30:4488-4493.

4. Zhao L, Hu L, Huo K, Zhang Y, Wu Z, Chu PK. Mechanism of cell repellence on quasi-aligned nanowire arrays on Ti alloy. Biomaterials. 2010;31:8341-8349.

5. Soppimath KS, Aminabhavi TM, Kulkarni AR, Rudzinski WE. Biodegradable polymeric nanoparticles as drug delivery devices. J Control Release. 2001;70:1-20.

6. Liu B, Khare A, Aydil ES. $\mathrm{TiO}_{2}-\mathrm{B}$ /anatase core-shell heterojunction nanowires for photocatalysis. ACS Appl Mater Interfaces. 2011;3: 4444-4450.

7. Kim W, Ng JK, Kunitake ME, Conklin BR, Yang P. Interfacing silicon nanowires with mammalian cells. J Am Chem Soc. 2007;129: 7228-7229.

8. Liu Y, Wang XL, Yang F, Yang XR. Excellent antimicrobial properties of mesoporous anatase $\mathrm{TiO}_{2}$ and $\mathrm{Ag} / \mathrm{TiO}_{2}$ composite films. Microporous Mesoporous Mater. 2008;114:431-439.

9. Dong W, Zhang T, Epstein J, et al. Multifunctional nanowire bioscaffolds on titanium. Chem Mater. 2007;19:4454-4459.

10. Li S, Ni J, Liu X, et al. Surface characteristics and biocompatibility of sandblasted and acid-etched titanium surface modified by ultraviolet irradiation: an in vitro study. J Biomed Mater Res B Appl Biomater. 2012;100:1587-1598.

11. Feng X, Zhu K, Frank AJ, Grimes CA, Mallouk TE. Rapid charge transport in dye-sensitized solar cells made from vertically aligned single-crystal rutile $\mathrm{TiO}_{2}$ nanowires. Angew Chem Int Ed Engl. 2012;51:2727-2730. 
12. Feng X, Shankar K, Varghese OK, Paulose M, Latempa TJ, Grimes CA. Vertically aligned single crystal $\mathrm{TiO}_{2}$ nanowire arrays grown directly on transparent conducting oxide coated glass: synthesis details and applications. Nano Lett. 2008;8:3781-3786.

13. Kandori K, Tsuyama S, Tanaka H, Ishikawa T. Protein adsorption characteristics of calcium hydroxyapatites modified with pyrophosphoric acids. Colloids Surf B. 2007;58:98-104.

14. Wu S, Liu X, Hu T, et al. A biomimetic hierarchical scaffold: natural growth of nanotitanates on three-dimensional microporous Ti-based metals. Nano Lett. 2008;8:3803-3808.

15. Aita H, Hori N, Takeuchi M, et al. The effect of ultraviolet functionalization of titanium on integration with bone. Biomaterials. 2009;30 1015-1025.

16. Att W, Hori N, Takeuchi M, et al. Time-dependent degradation of titanium osteoconductivity: an implication of biological aging of implant materials. Biomaterials. 2009;30:5352-5363.

17. Lee J, Kang BS, Hicks B, et al. The control of cell adhesion and viability by zinc oxide nanorods. Biomaterials. 2008;29:3743-3749.

18. Arnold M, Cavalcanti-Adam EA, Glass R, et al. Activation of integrin function by nanopatterned adhesive interfaces. Chemphyschem. 2004;5:383-388.

19. Giancotti FG. A structural view of integrin activation and signaling. Dev Cell. 2003;4:149-151.
20. Cavalcanti-Adam EA, Volberg T, Micoulet A, Kessler H, Geiger B, Spatz JP. Cell spreading and focal adhesion dynamics are regulated by spacing of integrin ligands. Biophys J. 2007;92:2964-2974.

21. Cavalcanti-Adam EA, Micoulet A, Blummel J, Auernheimer J, Kessler H, Spatz JP. Lateral spacing of integrin ligands influences cell spreading and focal adhesion assembly. Eur J Cell Biol. 2006;85:219-224.

22. Biggs MJ, Richards RG, Dalby MJ. Nanotopographical modification: a regulator of cellular function through focal adhesions. Nanomedicine. 2010;6:619-633.

23. Mata A, Su X, Fleischman AJ, et al. Osteoblast attachment to a textured surface in the absence of exogenous adhesion proteins. IEEE Trans Nanobioscience. 2003;2:287-294.

24. Wang C, Gong Y, Zhong Y, Yao Y, Su K, Wang DA. The control of anchorage-dependent cell behavior within a hydrogel/microcarrier system in an osteogenic model. Biomaterials. 2009;30:2259-2269.

25. Gtigoriou V, Shapiro IM, Cavalcanti-Adam EA, Composto RJ, Ducheyne P,Adams CS. Apoptosis and survival of osteoblast-like cells are regulated by surface attachment. J Biol Chem. 2005;280:1733-1739.

26. Luo Y, Shoichet MS. A photolabile hydrogel for guided threedimensional cell growth and migration. Nat Mater. 2004;3:249-253.

27. Yang Y, Cavin R, Ong JL. Protein adsorption on titanium surfaces and their effect on osteoblast attachment. Biomed Mater Res A. 2003;67: 344-349. 


\section{Supplementary figures}

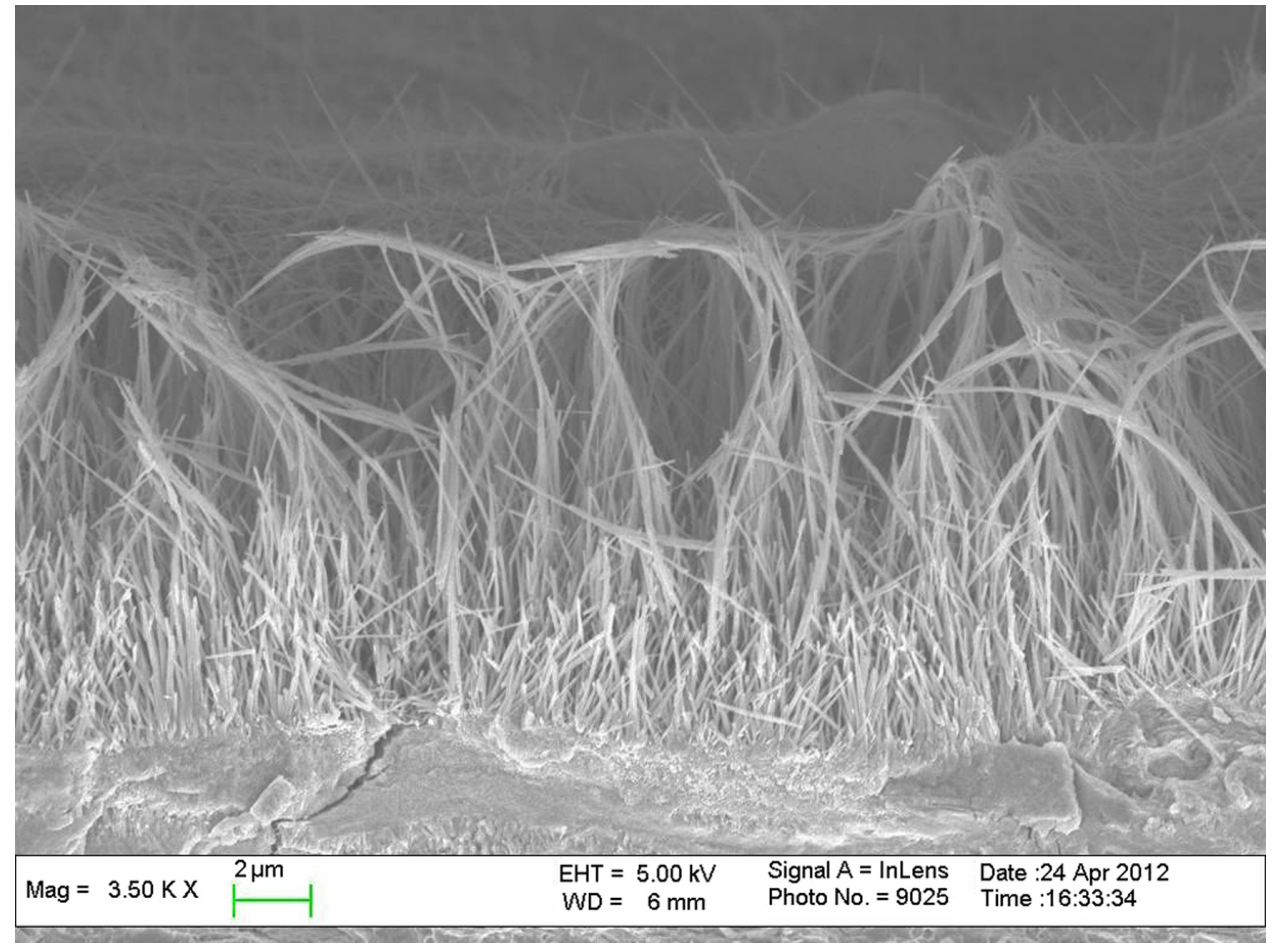

Figure SI Field emission scanning electron microscopic images of titanate nanowire scaffolds, cross-sectional view.
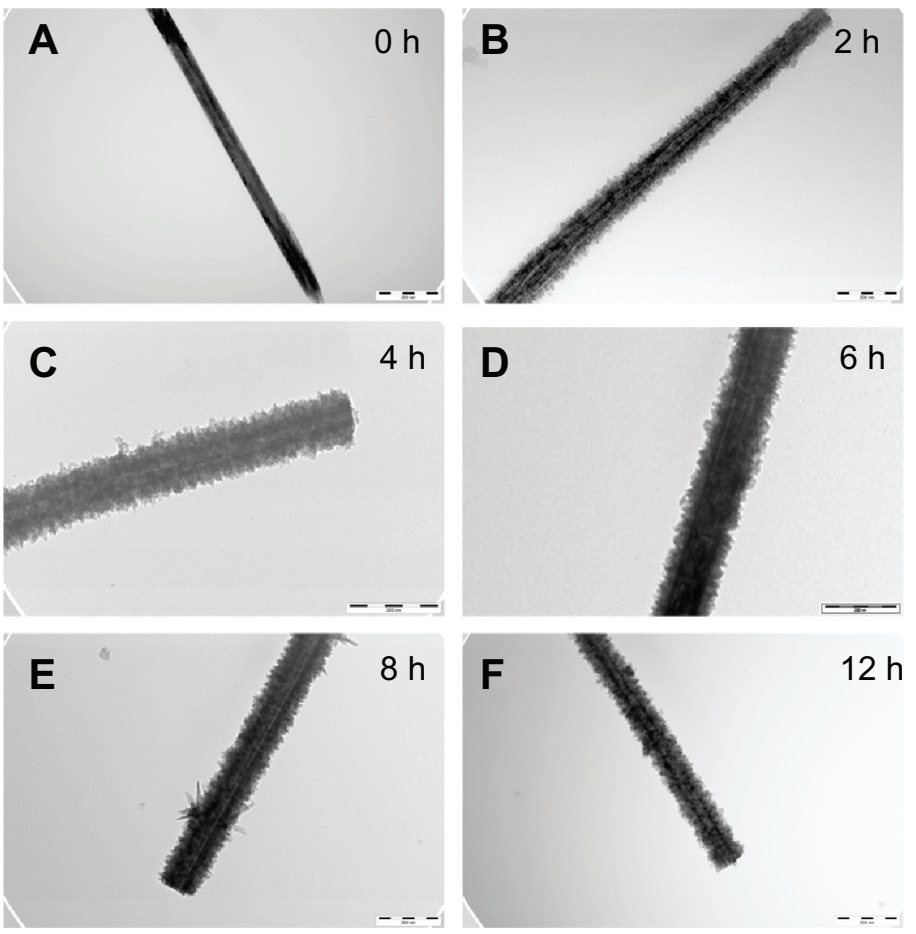

Figure S2 Transmission electron micrographs of titanate nanowire scaffolds decorated with anatase, prepared by treating nanowires in TiCl 4 solution for (A) 0 , (B) 2 , (C) 4, (D) 6, (E) 8, and (F) 12 hours. Note: Scale bar $200 \mathrm{~nm}$ 

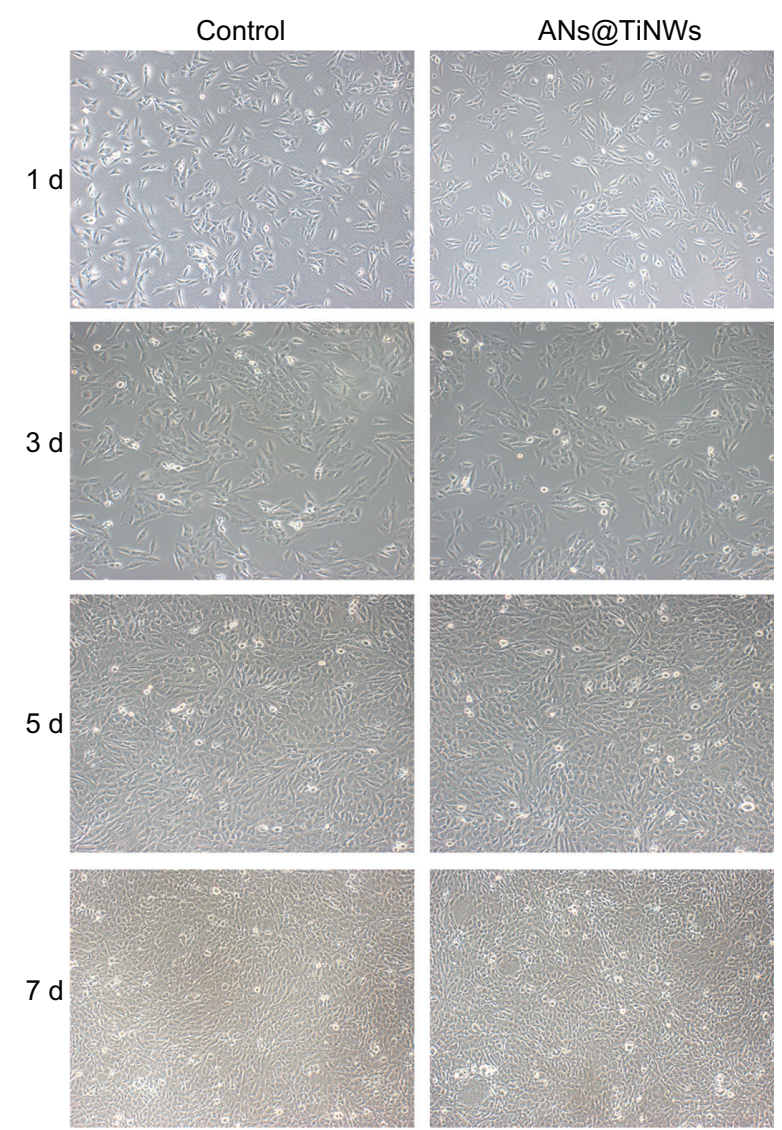

Figure S3 Material toxicity test.

Abbreviation: ANs@TiNWs, titanate nanowire scaffolds decorated with anatase.

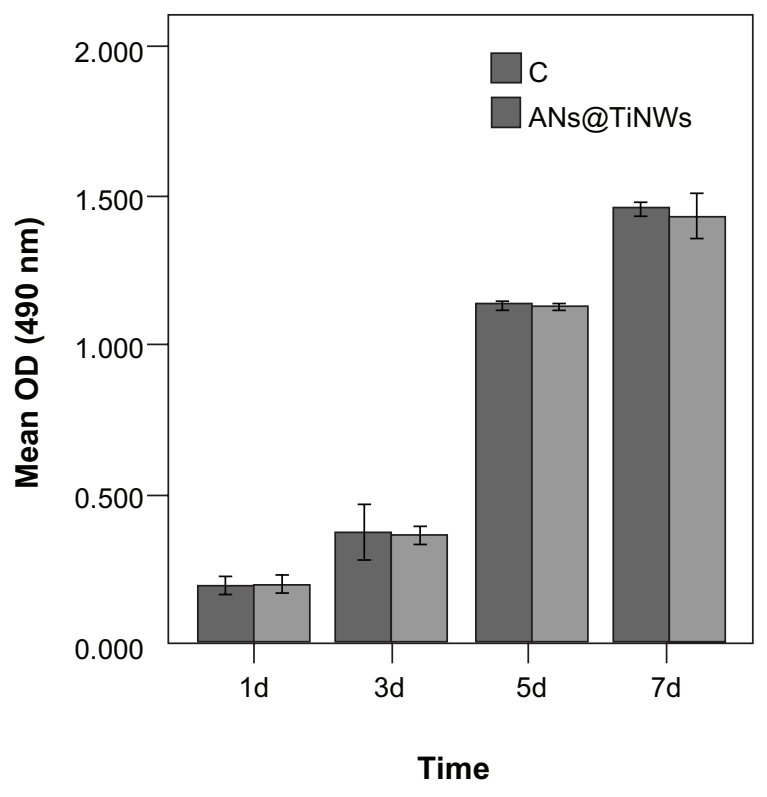

Figure S4 Mean ( \pm standard deviation) optical density calculated from Figure S3. Abbreviations: ANs@TiNWs, titanate nanowire scaffolds decorated with anatase; C, controls; OD, optical density.
International Journal of Nanomedicine

\section{Publish your work in this journal}

The International Journal of Nanomedicine is an international, peerreviewed journal focusing on the application of nanotechnology in diagnostics, therapeutics, and drug delivery systems throughout the biomedical field. This journal is indexed on PubMed Central, MedLine, CAS, SciSearch $₫$, Current Contents ${ }^{\circledR} /$ Clinical Medicine,

\section{Dovepress}

Journal Citation Reports/Science Edition, EMBase, Scopus and the Elsevier Bibliographic databases. The manuscript management system is completely online and includes a very quick and fair peer-review system, which is all easy to use. Visit http://www.dovepress.com/ testimonials.php to read real quotes from published authors.

\footnotetext{
Submit your manuscript here: http://www.dovepress.com/international-journal-of-nanomedicine-journal
} 